\title{
Welcome to the journal of Energy Materials: a new scientific journal
}

\author{
Yuping $\mathrm{Wu}^{1}$, Bin $\mathrm{Zhu}^{2}$ \\ 1School of Energy Science and Engineering, Nanjing Tech University, Nanjing 210014, Jiangsu, China. \\ ${ }^{2}$ School of Energy and Environment, Southeast University, Nanjing 211189, Jiangsu, China.
}

Correspondence to: Prof. Yuping Wu, School of Energy Science and Engineering, Nanjing Tech University, Nanjing 210014, Jiangsu, China. E-mail: wuyp@fudan.edu.cn; Prof. Bin Zhu, China \& School of Energy and Environment, Southeast University, Nanjing 211189, Jiangsu, China. E-mail: zhu-bin@seu.edu.cn

How to cite this article: Wu Y, Zhu B. Welcome to the journal of Energy Materials: a new scientific journal. Energy Mater 2021;1:100001. https://dx.doi.org/10.20517/energymater.2021.01

Received: 2 May 2021 Accepted: 2 May 2021 Available online: 7 May 2021

Academic Editors: Yuping Wu, Bin Zhu Copy Editor: Whitney Xu Production Editor: Xi-Jun Chen

The energy materials have emerged over the last decade showing the importance of research and development, covering various forms of the organic, and inorganic substances and composites from microto nano-structures, zero dimension to three dimensions, experimental and theoretical fundamentals and applications, with emphasis on functions and device performances, in particularly for energy conversion and storage applications, typically, battery and fuel cell, as well as solar cell, electrolysis, water splitting, super capacitor, etc. We often find the existing materials and properties used for these energy applications in a new way or with a new surprise, which hints a need for us to rediscover their properties and reunderstand their natures. For example, existing electrode materials for ceramic batteries and fuel cells are commonly semiconducting nature, but they are rarely investigated to combine with this nature and properties, e.g., band and band structure for the electrochemical performance and process/mechanism.

Semiconductor materials and relevant physics have been focused by the photocatalyst, solar cell, especially perovskite solar cell fields, which have recently been extended widely to ceramic battery and fuel cell as an emerging hot area of the material families. Semiconductors and derived heterostructures with electronic (hole) conducting materials have been strongly developed with much better ionic conductors instead of a conventional ionic electrolyte. They have exhibited not only excellent material functions, and device performances, but also new fundamental and science. Furthermore, semiconductor electrochemistry is 
growing as a frontier understanding of the electrochemical energy devices, where energy band, band alignment, junction and built-in-field play a significantly important role in understanding the material functionality and device performance in research and development. The new movement indicates by introducing the semiconductors and physics into electrochemistry, many new materials, function designs and device technologies are emerging. Energy band structures and alignments, band bending and built-in electric field are all important parameters in this context to accomplish the necessary battery and fuel cell functionalities. It provides new ideas and new solutions to the problems beyond conventional electrochemistry and presents new inter-disciplinary approaches to develop clean energy conversion and storage technologies for our sustainable society.

At the same time, a better understanding of the energy devices and scientific mechanisms in both materials and devices are progressing. In addition, nanomaterials and nano-device understandings are potential to continuously enhance and improve the material functionalities and device performances. Taking them as examples to give us a comprehensive view of current research frontiers with emphases on semiconductors and band theories applications in materials and devices/technologies as well as fundamental sciences, followed by all energy applications, battery, fuel cell, photoelectrolysis or photocatalysts, solar cells, metalair batteries, electrolyzers, etc. can be crosslinked based on the common principle and understanding.

The endeavors should look forward more openly to predicting a wider range of materials and new technologies and applications across the different disciplines of materials, technology, engineering, physics, and chemistry/electrochemistry. This is a cross-think and philosophy beyond the existing knowledge, which can stimulate more innovations and creations not only for future research and development on the energy material and technology, but also widely for new advanced energy conversion and storage applications.

The field of the energy materials exhibits strongly no discipline boundary but with interdisciplinary success. It can complement and strongly push the energy materials and devices to new types for practical uses and at the same time new materials and science to be discovered and new knowledge to be developed as a prosperous field so that a sustainable society can be well established.

The journal of Energy Materials provides an online platform for sharing of research data, new methodologies, mini/reviews, and perspectives on these exciting areas of research, involving partnerships between the academia and industry to solve the challenges of global energy crisis. At the same time, the journal supports publication of strong basic research which enables development of energy applications.

Energy Materials will be published bimonthly with additional special issues focusing on specific topics. We have assembled an eminent group of international researchers with expertise across the scopes covering energy materials for various electrochemical applications to serve on the Editorial Board. For manuscripts submitted to Energy Materials, scientific excellence and originality will be the criteria for acceptance. We also aim to provide fast processing time for articles submitted for publication. In this first issue of Energy Materials, it is scheduled to be launched in October 2021. We would like to invite you to submit papers for future issues of Energy Materials (https://oaemesas.com/EnergyMater).

\section{DECLARATIONS}

\section{Authors' contributions}

Preparing the manuscript draft: Wu Y, Zhu B 


\section{Availability of data and materials}

Not applicable.

\section{Financial support and sponsorship}

The author has not declared a specific grant for this editorial from any funding agency in the public, commercial, or not-for-profit sectors.

\section{Conflicts of interest}

Both authors declared that there are no conflicts of interest.

\section{Ethical approval and consent to participate}

Not applicable.

\section{Consent for publication}

Not applicable.

\section{Copyright}

(c) The Author(s) 2021. 